\title{
TIPOLOGIA, GENESIS Y DESARROLLO DE LAS HELADAS EN EL VALLE MEDIO DEL EBRO
}

\author{
$M^{\text {a }}$ Luz HERNANDEZ \\ Departamento de Geografía y Ordenación del Territorio \\ Universidad de Zaragoza
}

Resumen: Este trabajo tiene como objetivo el análisis de las situaciones de tiempo que producen heladas en el valle medio del Ebro, conocer su tipología, su duración y los valores que presentan determinadas variables climáticas como la presión, la temperatura, la humedad relativa y el viento, de cara a aumentar la eficacia y rentabilidad en la lucha contra las heladas. Para ello se analizan las situaciones de tiempo que produjeron temperaturas iguales o inferiores a $0^{\circ}$ en el observatorio del Aeropuerto de Zaragoza (período 1972-86) durante todo el período frío y en los meses de primavera.

La conclusión que extrae es que las heladas más frecuentes son las de irradiación, siendo la influencia de la topografía casi tan importante como la evolución atmosférica, en la génesis de las heladas en el valle medio del Ebro. En definitiva, las heladas más rigurosas en conjunto son las que presentan un origen doble o combinado, con entrada de una advección fría y posterior estabilización de la masa de aire.

Palabras clave: Heladas, tipología, valle medio del Ebro.

Abstract: This paper analyses the weather of the situations of frost, her typology, duration and the values of pressure, temperature, relative humidity and wind at Zaragoza Airport (Ebro valley) (1972-86), to improve frost prediction. The most frecuency cause is irradiation and the lowest temperatures are generated by a cold advent and later stabilization of the air.

Key words: Frost, typology, middle Ebro valley 


\section{INTRODUCCION}

Existe una intrínseca e íntima relación entre la evolución de las temperaturas y el desarrollo de la actividad vegetativa de las plantas. Y si bien es cierto que éstas no son el único factor que determina el ritmo de la vida vegetal es innegable que se trata, al menos, del más aparente. El descenso de los valores térmicos condiciona la parada vegetativa por frío, en la que las plantas van desarrollando mecanismos de defensa que mantendrán hasta que, a finales de invierno-comienzos de la primavera, reinicien el despertar del letargo invernal.

Este punto de partida pone de relieve el papel que juegan las temperaturas. Resulta imprescindible que los valores térmicos sean lo suficientemente bajos (inferiores a $7^{\circ}$ ) durante los inviernos a fin de que se completen las necesidades de frío de las plantas en estado latente. En el caso de los cultivos frutales son imprescindibles de cara a la correcta floración y cuajado de los frutos, según los umbrales correspondientes a las diferentes especies y variedades, con el consiguiente efecto negativo que puede suponer la no satisfacción de esta condición.

Pero con la llegada del ascenso térmico y pérdida de las defensas desarrolladas contra las bajas temperaturas, la aparición del frío se convierte en una amenaza y en un peligro contra los vegetales. En el caso de los cultivos, los daños que las heladas producen se traducen no sólo en daños fisiológicos o mecánicos, sino también económicos y consiguientemente sociales. De ahí el interés que reside en los estudios de climatología aplicada, enfoque desde el que se realiza este trabajo.

Así, los efectos producidos por las heladas pueden resultar perjudiciales dependiendo de tres factores: de su duración, de su intensidad, pero sobre todo del momento del ciclo vegetativo en que se producen y del estado fenológico de las plantas en ese momento dado. Por ello, para luchar contra las heladas es necesario, en primer lugar, caracterizar su tipología y desarrollo. Este conocimiento permite la instalación y puesta en marcha de los métodos de defensa que más eficaces y económicos pueden resultar en función de la problemática existente.

Desde esta premisa este trabajo tiene como objetivo el análisis de las situaciones de tiempo que producen heladas en un área de trabajo determinada que es el valle medio del Ebro, conocer su tipología en función de las situaciones de tiempo a las que se presentan asociadas, su duración, si son más frecuentes unas u otras en determinados momentos del año y los valores que presentan determinadas variables climáticas como la presión, la temperatura, la humedad relativa y el viento. 
Este es el punto de partida para analizar posteriormente la intensidad de las heladas, su probabilidad y frecuencia de aparición y el riesgo de que produzcan daños sobre las cosechas (HERNANDEZ, en prensa).

\section{CONCEPTO DE HELADA}

Se considera que se ha producido helada climática a partir del momento en el que el termómetro desciende a $0^{\circ 1}$, aunque los valores térmicos que se miden en los observatorios corresponden a los del aire, con lo que las diferencias que pueden existir entre éstos y los del suelo -ligeramente superiores en función de su albedo- o los de los diferentes órganos de las plantas pueden ser notables. GARCIA DE PEDRAZA (1978) ejemplifica este gradiente vertical señalando que la temperatura puede ser de $-6^{\circ}$ a 10 $\mathrm{cm}$. del suelo, y de $0^{\circ}$ a $150 \mathrm{~cm}$. Ello nos indica que las plantas herbáceas, como cereales y hortalizas entre los cultivos, sufren valores térmicos bastante inferiores a los de la temperatura medida en caseta, mientras que las de los arbustos y árboles de pequeño tamaño, como son los árboles frutales, se encuentran en una situación bastante similar a la de la temperatura del aire, que es la más empleada en los estudios de climatología aplicada. Por tanto, sería muy conveniente poder disponer de los índices actinotérmicos ${ }^{2}$ con objeto de afinar más el conocimiento de las condiciones realmente soportadas por los cultivos en los períodos críticos al desarrollo de las mismos en función de la temperatura.

De cualquier forma, estas diferencias suelen ser menos acusadas cuando la génesis de las bajas temperaturas está ligada a la invasión de una masa fría, puesto que la constante renovación del aire homogeneiza los valores térmicos que se registran; por el contrario, estas diferencias son más patentes cuando las heladas se producen con una situación estable, anticiclónica, con vientos en calma. Las variaciones se producen entonces a una escala más local, con lo que además son más difíciles de prevenir y por lo tanto de aplicar medidas de lucha contra las mismas.

1 En garita ventilada colocada a $150 \mathrm{~cm}$. del suelo (OMM, 1983).

2 Los índices actinotérmicos se definen como los valores de temperatura a diferentes distancias del suelo, generalmente $5,10,15 \ldots$ hasta $50 \mathrm{~cm}$. de altura. 


\section{TIPOS DE HELADA}

Por su origen, las heladas se pueden clasificar en tres tipos: de advección, de irradiación y de evaporación.

\section{Heladas de advección}

Se presentan asociadas a la penetración de una masa de aire frío -polar-o muy frío -ártica- que provoca un descenso generalizado de las temperaturas en un radio de acción de gran escala. La procedencia del flujo frío puede ser doble: o bien acceder desde Noroeste, y ser por ello húmeda, o bien del centro de Europa o de Siberia, del Noreste, algo más seca por su trayectoria continental. A la entrada de estas masas de aire es a lo que se denomina "olas de frío" y suelen llegar acompañadas por fuertes vientos y con frecuencia provocan nubosidad y precipitaciones, en ocasiones en forma de nieve, más abundantes si han atravesado el Océano. Sus efectos se dejan sentir en el conjunto de la Península Ibérica o al menos en buena parte de ella, sin embargo su probabilidad de aparición no es demasiado alta. GARCIA DE PEDRAZA (1978) calcula el periodo de retorno en una cifra superior a los 30 años.

\section{Heladas de irradiación}

Las heladas de irradiación se presentan asociadas a una situación sinóptica anticiclónica que estabiliza la masa de aire que se encuentra sobre la superficie terrestre y permite la pérdida de calor durante la noche en forma de energía de onda larga -que no puede ser absorbida por los cuerpos, vegetales o animales, situados en la biosfera-, formando junto al suelo una inversión de temperatura que refuerza la estabilidad atmosférica, de forma que puede haber grandes variaciones de temperatura según la vertical en cortos intervalos. Este proceso se agudiza en áreas deprimidas topográficamente, como grandes hondonadas o vallonadas, lo que explica que presenten un carácter más local que las anteriores.

La Figura 1 representa el balance térmico entre la tierra y la atmósfera durante el día y la noche. Durante el día, la radiación solar directa, aun debilitada por la reflexión de las nubes, absorción de la atmósfera y la energía disipada, más la radiación difusa, superan ampliamente a la radiación terrestre y a otras pérdidas de calor por reflexión, evaporación, calor sensible y conducción. El balance térmico positivo motiva el calentamiento del suelo con flujo de calor hacia el interior de la tierra, que almacena ese calor. Durante la noche, la radiación solar desaparece y, aunque la tierra puede recibir calor procedente de la radiación atmosférica, calor sensible, conducción, y condensación del vapor de agua en forma de rocío, las pérdidas por evaporación $\mathrm{y}$, sobre todo, por radiación terrestre son tan fuertes que superan 
ampliamente los aportes, motivando un balance térmico negativo. En consecuencia, la tierra se enfría, estableciéndose un flujo de calor desde su interior a la superficie, que lo va irradiando a la atmósfera con lo que las temperaturas mínimas se observan de madrugada.

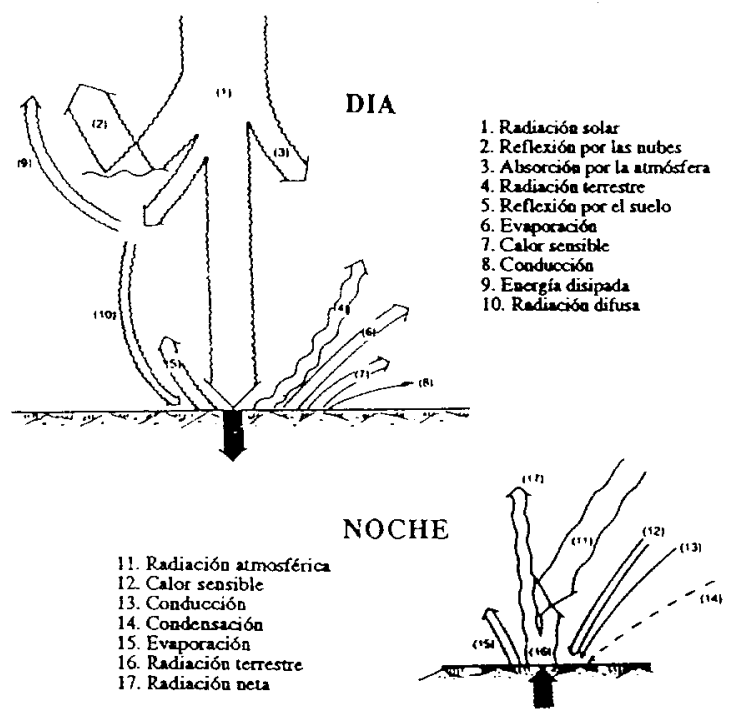

Figura 1.- Balance térmico tierra-atmósfera durante el día y la noche (URBANO, 1989).

Se puede afirmar que la intensidad de la radiación terrestre se sitúa, durante las noches de primavera, en cifras próximas a $l o s 0.5 \mathrm{cal} / \mathrm{cm}^{2} \mathrm{~min}$., así que las pérdidas de calor serán la diferencia entre esta radiación y los restantes factores del balance térmico, en el que juegan un papel muy importante tanto la radiación atmosférica como los procesos de evaporación y condensación.

Por otra parte, la diafanidad de la atmósfera es un factor que favorece la génesis de heladas de irradiación, puesto que la radiación terrestre, por su larga longitud de onda, se pierde en las capas altas de la atmósfera a menos que existan en ella partículas que, interceptando la radiación, puedan reflejarla. El vapor de agua, al polvo y el humo atmosférico actúan como pantallas y devuelven a la tierra parte de la radiación emitida, reduciendo la velocidad de enfriamiento. El poder máximo lo presentan aquellas partículas de color brillante y diámetro similar a la longitud de onda de la radiación (10 $\mu$ aprox.). 
En definitiva, las pérdidas de calor por radiación neta durante la noche se sitúan en las proximidades de $0.1 \mathrm{cal} / \mathrm{cm}^{2} \mathrm{~min}$ para suelos desnudos, con bajo contenido de humedad atmosférica (50-60\%) y temperatura del suelo entre 0 y $10^{\circ}$. Es conveniente destacar que el valor de la radiación neta en el suelo desnudo aumenta con la temperatura y disminuye al aumentar la humedad relativa.

Por otra parte, la presencia o no de vegetación modifica la probabilidad de aparición de heladas mediante este proceso de irradiación nocturna. En los suelos cubiertos de vegetación el riesgo de heladas es mayor que en los desnudos, ya que la vegetación presenta una superficie receptora y radiante enorme. Además, las plantas de mayor superficie foliar son los que presentan un riesgo de enfriamiento por radiación más acusado.

El papel que juega el suelo también es importante. Y son factores edáficos de interés el color, el calor específico, la estructura, conductividad térmica y el estado de humedad, principalmente. Los suelos oscuros tienen mayor capacidad para absorber la radiación solar y, en consecuencia, se calientan más durante el día. La pérdida de calor por radiación es mayor en los suelos oscuros, pero el nivel de enfriamiento dependerá de los restantes factores del balance térmico. El calor específico del suelo depende al mismo tiempo de su textura, volumen de poros y grado de humedad, pero mientras que el efecto de la textura es poco importante, sí lo es el de la humedad. Los suelos húmedos necesitan mayor aporte de calor para elevar su temperatura, pero presentan mayor inercia térmica y tardan más en enfriarse una vez calientes. Por otra parte, un suelo recién labrado se enfría con mayor rapidez que un suelo apelmazado, puesto que en el primer caso existe mayor superficie expuesta a perder el calor por irradiación. Además, la paja, hierba seca o estiércol sobre el suelo suministran un buen aislamiento que disminuye la irradiación del suelo (FUENTES, 1978).Esta misma técnica de trabajo puede ser importante para reducir la tasa de evapotranspiración (URBANO, 1989).

Por todo ello, las heladas por irradiación pueden ocasionar estragos en los cultivos, sobre todo las que se producen en otoño y primavera, puesto que entonces las temperaturas diurnas suelen ser lo suficiente elevadas como para que las plantas se encuentren ya en el período de actividad vegetativa.

\section{Heladas de evaporación}

Son debidas a que el agua depositada sobre las plantas, al evaporarse rápidamente, roba de ellas el calor de evaporación y las enfría con brusquedad, por lo que la temperatura de algunos órganos vegetales desciende hasta valores negativos. La cantidad de calor necesaria para vaporizar el agua líquida a la temperatura ambiente es tan grande ( 600 calorías por gramo de agua) que puede provocar estos 
descensos de temperatura tan importantes. Cuando este proceso se realiza sobre órganos delicados de la planta, como yemas, flores, o pequeños frutos, puede originarse un enfriamiento suficiente para que tales órganos sean dañados.

Este tipo de heladas se produce cuando un frente frío o un descenso marcado de las temperaturas origina una condensación importante del vapor de agua atmosférico en forma de rocío provocando una reducción notable de la humedad relativa. Pasado este frío, al restablecerse las temperaturas normales, se producirá un gradiente de tensión de vapor muy fuerte entre las superficies vegetales cubiertas de agua y la atmósfera que se ha desecado. El calor necesario para vaporizar el agua líquida es sustraido de las superficies vegetales provocando su enfriamiento.

Así pues, este tipo de heladas puede presentarse de dos formas: generadas por una situación de borrasca o en presencia de estabilidad atmosférica. En el primer caso el paso de un frente frío provoca precipitaciones que humedecen las plantas; ese agua se evapora rápidamente al llegar vientos fríos y secos con baja humedad relativa. Con condiciones estables suelen formarse condensaciones sobre las plantas, en forma de rocío o escarcha, según la temperatura ambiental, que se evaporan con la salida del sol, robando calor a esas plantas y registrándose heladas.

\section{SITUACIONES QUE GENERAN HELADAS EN EL VALLE MEDIO DEL EBRO}

Se han analizado las situaciones sinópticas que han generado cada una de las heladas registradas en el observatorio de Aeropuerto de Zaragoza, como estación más representativa del sector central de la Depresión del Ebro, en el período de 15 años comprendido entre 1972 y 1986, a partir del estudio del Boletín Meteorológico Diario. Los valores de las variables climáticas (temperatura, humedad relativa, velocidad del viento) proceden del banco de datos diario de esta estación -la única completa en este sector- facilitados por el Instituto Nacional de Meteorología (Centro Zonal del Ebro). Se ha analizado la cartografía de la atmósfera en superficie y la topografía de la superficie de $500 \mathrm{mb}$ a las $12 \mathrm{~h}$. de cada uno de los días en los que se registró una temperatura igual o inferior a $0^{\circ}$. Para su clasificación se ha tenido en cuenta la situación de la atmósfera en el sector del Valle del Ebro, que en numerosas ocasiones coincide también con el resto de la Península, aunque no siempre pueda ser asimilable, sobre todo con las áreas más alejadas de la zona de estudio. De la combinación de ambos mapas se ha establecido la tipología de tipos de tiempo asociados a helada ${ }^{3}$.

3 De ahora en adelante las situaciones de tiempo expresadas en dos términos indican: (situación de superficie)+(situación de las capas altas de la atmósfera). 
Los resultados obtenidos del análisis de todas las situaciones de tiempo que han dado origen a una temperatura igual o inferior a $0^{\circ}$ están reflejados en los gráficos y tablas siguientes:

Tabla 1.- Situaciones de tiempo que han generado heladas en el observatorio de Zaragoza Aeropuerto (período 1972-86).

Sit. $\mathrm{n}^{\circ}$ veces $\%$ tmedia Tmedia HRmax HRmin HRmed Vel.viento

$\begin{array}{lrrrrrrrr}\mathrm{A} & 243 & 67.1 & -1.9^{\circ} & 8.8^{\circ} & 91 & 66 & 78 & 6 \\ \mathrm{~A}+\mathrm{N} & 2 & 0.5 & -2.8^{\circ} & 2.7^{\circ} & 83 & 72 & 78 & 9 \\ \mathrm{~A}+\mathrm{NE} & 14 & 3.9 & -2.2^{\circ} & 8.9^{\circ} & 81 & 52 & 67 & 12 \\ \mathrm{~A}+\mathrm{NW} & 6 & 1.6 & -1.8^{\circ} & 11.5^{\circ} & 86 & 55 & 70 & 8 \\ \mathrm{~A}+\mathrm{SW} & 2 & 0.5 & -1.5^{\circ} & 6.8^{\circ} & 100 & 94 & 97 & 7 \\ \mathrm{~A}+\mathrm{W} & 2 & 0.5 & -1.0^{\circ} & 11.6^{\circ} & 98 & 63 & 81 & 4 \\ \mathrm{~N} & 9 & 2.5 & -1.9^{\circ} & 6.0^{\circ} & 76 & 47 & 61 & 12 \\ \mathrm{NE} & 34 & 9.4 & -1.7^{\circ} & 5.8^{\circ} & 72 & 50 & 61 & 20 \\ \mathrm{NW} & 16 & 4.4 & -1.3^{\circ} & 10.3^{\circ} & 83 & 54 & 68 & 11 \\ \mathrm{~W} & 3 & 0.8 & -0.4^{\circ} & 9.4^{\circ} & 75 & 61 & 68 & 9 \\ \mathrm{SW} & 1 & 0.3 & -0.3^{\circ} & 2.0^{\circ} & 96 & 92 & 94 & 0 \\ \mathrm{~B} & 17 & 4.7 & -2.5^{\circ} & 7.0^{\circ} & 85 & 62 & 74 & 13 \\ \text { otras }^{4} & 13 & 3.6 & -1.2^{\circ} & 9.1^{\circ} & 82 & 56 & 69 & 9\end{array}$

$\mathrm{t}=$ temp. media de las mínimas diaria; $\mathrm{T}=$ temp. media de las máximas diaria HRmax; HRmin; HRmed = hum.rel.máxima, mínima y media diarias, en \% vel.viento $=$ velocidad media diaria del viento, a $0 \mathrm{~h}$., en $\mathrm{km} / \mathrm{h}$, a $2 \mathrm{~m}$ de altura Fuente: I.N.M. Elaboración propia

El análisis de los datos nos permite conocer que el mayor porcentaje de heladas registradas en el centro del valle del Ebro están ocasionadas por una situación de estabilidad atmosférica, más intensa y perceptible en las capas bajas en contacto con la superficie, aunque puedan verse alimentadas por la entrada de aire frío -de componente norte- en altura. El total de situaciones anticiclónicas que dieron lugar a estas temperaturas bajas, a lo largo de los 15 años de estudio, fueron 269, lo que supone un $74.3 \%$ de las analizadas. Dentro de ellas, las más frecuentes siguen siendo las definidas por estabilidad en toda la atmósfera, generalmente afectadas por un anticiclón de gran radio de acción.

\footnotetext{
4 Pantano barométrico y otras situaciones indefinidas.
} 


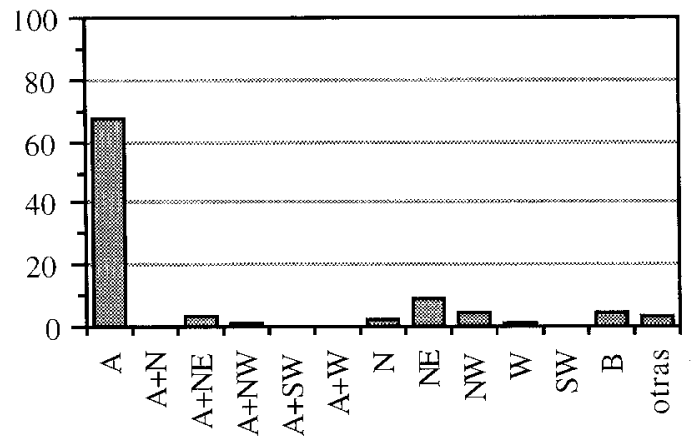

Fuente: I.N.M. Elaboración propia

Figura 2.- Tipos de tiempo asociados a heladas. Valores porcentuales.

Estas situaciones se caracterizan por presentar valores altos de presión en superficie, superiores a los $1.020 \mathrm{mb}$, y la temperatura de las capas altas oscila en torno a los $-20^{\circ} /-24^{\circ}$. Tanto en invierno como en primavera esta disposición da origen a heladas de irradiación, a las que en alguna ocasión suelen acompañar las nieblas, tan persistentes durante los meses de invierno en la depresión del Ebro, que impiden una disminución aún mayor de la temperatura. No obstante en algunas ocasiones también se producen heladas cuando la temperatura de las capas altas de la atmósfera es inferior a $-20^{\circ}$, pero entonces la presión en superficie es muy alta, con lo que se refuerza el efecto estabilizador y se generan igualmente heladas de irradiación.

En este mismo sentido se han analizado los valores de humedad relativa de los días en los que hubo helada (Tabla 1), pudiéndose constatar que tanto los valores de la mínima como de la máxima diaria son sensiblemente más altos cuando estas temperaturas bajas se asocian a estabilidad anticiclónica. El fuerte descenso térmico como consecuencia de la pérdida de calor nocturna genera este aumento de la humedad relativa. Ello provoca el que incluso con presencia de nieblas se registren heladas en este área, aunque este meteoro suela funcionar como moderador térmico interceptando la radiación y reflejándola. La alta humedad da también lugar a que se pueda formar escarcha sobre el suelo y sobre la superficie de los vegetales. En cualquier caso, desde el punto de vista agrícola, la presencia o no de hielo no interesa tanto como el saber que se ha producido una temperatura igual o inferior a $0^{\circ}$. DE FINA Y RAVELO (1979) indican que las plantas sufren un efecto pernicioso siempre que se producen heladas, independientemente de que se manifieste la presencia o no de un depósito de escarcha -las llamadas heladas blancas-. 
Las heladas asociadas a situaciones estables, además de ser las más frecuentes son también las que producen, en conjunto, los valores de temperatura más bajos, tanto en las mínimas como en las máximas. CAÑADA (1990), en su diferenciación de tipos de tiempo ciclónico y anticiclónico también señala que, en los valores de la temperatura del aire en superficie el grupo anticiclónico tiene valores más bajos en el invierno ${ }^{5}$. Este hecho se explica porque es precisamente durante los meses de diciembre, enero y febrero cuando la radiación recibida por las latitudes templadas del Hemisferio Norte es menor, además de ser el período en el que son más frecuentes las advecciones frías. Como consecuencia se registra un enfriamiento importante de la superficie terrestre, que se agudiza con la pérdida de calor por irradiación nocturna y que se suaviza cuando hay una mayor nubosidad, pues la capa de nubes actúa como una pantalla que frena la pérdida de calor, por ello el tipo ciclónico tiene temperaturas más altas. Y por esa razón las situaciones menos estables producen mucho menos frecuentemente heladas.

Este comportamiento, con entrada de una advección fría y posterior estabilización de la masa de aire y génesis de valores térmicos aún más bajos se repite frecuentemente en las situaciones de helada. Ello se puede comprobar también al analizar la velocidad media del viento de los días en los que se registró helada. Es frecuente encontrarse con la situación que se acaba de describir, en la que se produce la entrada de flujo de componente norte con vientos de velocidad moderada, de grado $4 \mathrm{o}$ superior de la escala de Beaufort, es decir, con velocidades superiores a los $20 \mathrm{~km} / \mathrm{h}$ a $2 \mathrm{~m}$. de altura del suelo. Cuando cesa la advección septentrional, el viento se encalma (grados 1 o 2 de esa escala). A partir de ese momento la temperatura desciende aún algo más respecto a los días precedentes.

Nuevamente en la Tabla 1 están reflejados los valores medios de la velocidad del viento en los días de helada, siendo sensiblemente superiores los que corresponden a los días dominados por una situación advectiva, sobre todo aquéllas de componente norte, destacando las que se presentan asociadas a la entrada de una masa de aire procedente del continente europeo, fría, que da origen también a temperaturas bajas y seca como lo demuestra el que se observen entonces los valores más bajos de humedad relativa, tanto máxima como mínima.

Por el contrario, los días de helada asociados estabilidad atmosférica presentan vientos en calma o semiencalmados, grados 0 o 1 de la escala de Beaufort. Las situaciones anticiclónicas se caracterizan por presentar una velocidad media del viento a las $0 \mathrm{~h}$. de $6 \mathrm{~km} / \mathrm{h}$. Además, de las 243 veces en que se produjo una

5 Sin embargo, primavera, verano y otoño caracterizan al tipo anticilónico con temperaturas más altas que al tipo ciclónico. 
temperatura inferior a los $0^{\circ}$ en el período de estudio 97 , el $40 \%$, observaron vientos totalmente en calma.

Otro aspecto analizado ha sido la duración media de los episodios de helada. Los 362 días de helada observados en el período 1972-86 se reparten entre 200 episodios fríos, lo que significa que cada uno de éstos está integrado por una media de 1.81 días, que se reparten como se indica en la Tabla 4, en función de su relación con las situaciones de tiempo que los han generado.

Tabla 2.- Duración media de los episodios de helada (período 1972-86).

\begin{tabular}{lrr} 
A & 107 & 2.3 \\
$\mathrm{~A}+\mathrm{N}$ & 2 & 1 \\
$\mathrm{~A}+\mathrm{NE}$ & 8 & 1.7 \\
$\mathrm{~A}+\mathrm{NW}$ & 6 & 1 \\
$\mathrm{~A}+\mathrm{SW}$ & 2 & 1 \\
$\mathrm{~A}+\mathrm{W}$ & 2 & 1 \\
$\mathrm{~N}$ & 7 & 1.3 \\
$\mathrm{~N}-\mathrm{NE}$ & 1 & 1 \\
$\mathrm{~N}-\mathrm{NW}$ & 1 & 1 \\
$\mathrm{NE}$ & 21 & 1.6 \\
$\mathrm{NW}$ & 14 & 1.1 \\
$\mathrm{~W}$ & 3 & 1 \\
$\mathrm{SW}$ & 1 & 1 \\
$\mathrm{~B}$ & 13 & 1.3 \\
otras & 13 & 1.13 \\
\hline
\end{tabular}

Fuente: I.N.M. Elaboración propia 


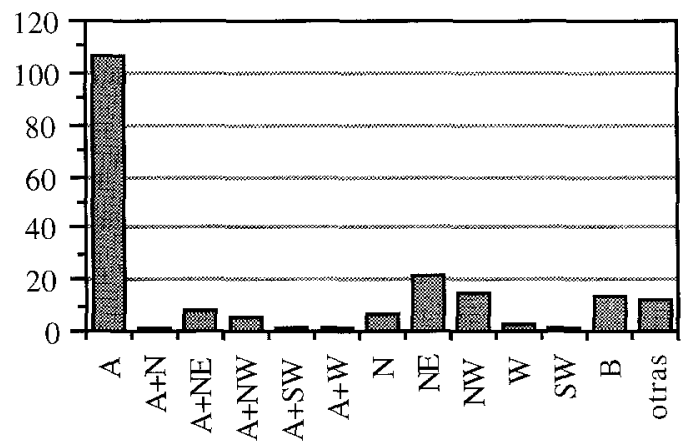

Fuente: I.N.M. Elaboración propia

Figura 3.- Número de episodios de helada asociados a cada situación.

El análisis de estos datos termina por confirmar la génesis de las heladas en el valle medio del Ebro ligadas preferentemente a situaciones de estabilidad y su calificación como heladas de irradiación, aunque también frecuentemente se vean apoyadas por la entrada de una masa de aire frío en los días anteriores a observarse las temperaturas bajas.

Por las especiales repercusiones bioclimáticas, económicas y sociales que presentan las heladas tardías se han analizado también las situaciones de tiempo que generaron las de los siguientes grupos de meses: las del conjunto de la primavera, de febrero, marzo y abril por un lado; y las de marzo y abril por otro. Se han tomado como referencia estos meses puesto que son las fechas en las que se producen los estados fenológicos de las plantas más sensibles a las heladas, siendo entonces más importantes los efectos económicos y biológicos de este fenómeno climático. A medida que la temperatura va ascendiendo y que como consecuencia de ello los vegetales recuperan su actividad vegetativa, la susceptibilidad a los posibles daños producidos por aquéllas aumenta. Así, las plantas resisten bien las bajas temperaturas invernales con umbrales de daños por helada entre los $20^{\circ}$ y los $30^{\circ}$ bajo cero, con lo que es muy poco probable que entonces se produzcan consecuencias negativas fisológicas y/o económicas en las plantas. Los umbrales de resistencia de las diversas especies a las temperaturas bajas de primavera disminuyen considerablemente Baste señalar que temperaturas entre -1 y $-5^{\circ}$ producen daños en los frutales con botón cerrado, entre -0.5 y $-3^{\circ}$ en el momento de plena floración y la resistencia es todavía menor cuando los frutos son jóvenes. Bastan temperaturas entre -0.5 y $-1.6^{\circ}$ en función de las diferentes especies (HERNANDEZ, en prensa). 
De esta forma, estas heladas de primavera, también llamadas heladas tardías, son las responsables del mayor porcentaje de pérdidas que sufre la agricultura debido a las temperaturas, fundamentalmente en las plantaciones de frutales. Por ello y desde hace varios años se ha intensificado la investigación en este campo, tanto desde el punto de vista agronómico como desde el bioclimático.

Se consideran, en primer lugar, como heladas de primavera las que se producen a partir del día 1 de febrero, puyesto que en función de la fenología de frutales especies más afectadas por las heladas tardías- en el valle medio del Ebro ${ }^{6}$ y según las conclusiones obtenidas en otros trabajos (HERNANDEZ, 1992) esta fecha la que marca el final del período de reposo y el reinicio de la actividad vegetativa.

Tabla 3.- Situaciones de tiempo que han generado heladas de primavera en el observatorio de Zaragoza Aeropuerto (período 1972-86) (meses de febrero, marzo y abril).

Sit. $\mathrm{n}^{\circ}$ veces $\% \mathrm{t}$ media $\mathrm{T}$ media HRmax HRmin HRmed Vel.viento

\begin{tabular}{lrrrrrrrr} 
A & 61 & 59.8 & $-1.8^{\circ}$ & $12.2^{\circ}$ & 86 & 49 & 68 & 5 \\
$\mathrm{~A}+\mathrm{NE}$ & 8 & 7.8 & $-1.9^{\circ}$ & $11.6^{\circ}$ & 79 & 45 & 62 & 14 \\
$\mathrm{~A}+\mathrm{NW}$ & 2 & 1.9 & $-0.8^{\circ}$ & $11.0^{\circ}$ & 71 & 39 & 55 & 9 \\
$\mathrm{NE}$ & 10 & 9.8 & $0.0^{\circ}$ & $7.3^{\circ}$ & 72 & 45 & 58 & 21 \\
$\mathrm{NW}$ & 6 & 5.9 & $-0.8^{\circ}$ & $11.2^{\circ}$ & 81 & 39 & 60 & 15 \\
$\mathrm{~W}$ & 1 & 0.9 & $-0.9^{\circ}$ & $12.0^{\circ}$ & 88 & 48 & 68 & 7 \\
$\mathrm{~B}$ & 8 & 7.8 & $-2.3^{\circ}$ & $9.3^{\circ}$ & 86 & 50 & 68 & 12 \\
otras & 6 & 5.9 & $-2.0^{\circ}$ & $9.8^{\circ}$ & 78 & 54 & 66 & 12 \\
\hline
\end{tabular}

$\mathrm{t}=$ temp. media de las mínimas diaria; $\mathrm{T}=$ temp. media de las máximas diaria

HRmax; HRmin; HRmed = hum.rel.máxima, mínima y media diarias, en \% vel.viento $=$ velocidad media diaria del viento, a $0 \mathrm{~h}$., en $\mathrm{km} / \mathrm{h}$, a $2 \mathrm{~m}$ de altura Fuente: I.N.M. Elaboración propia

\footnotetext{
6 Datos proporcionados por la Unidad de Pomología de la Estación Experimental de "Aula Dei", del C.S.I.C.
} 
En primer lugar es necesario señalar que el número de días en los que se producen heladas disminuye sensiblemente, pero siguen siendo las situaciones estables las que las generan en un porcentaje más alto de ocasiones.

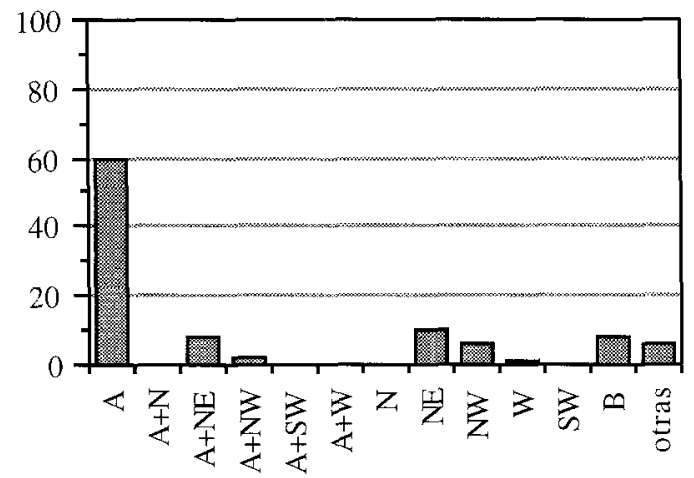

Fuente: I.N.M. Elaboración propia

Figura 4..- Tipos de tiempo asociados a helada en primavera (febrero-abril). Datos en $\%$

No obstante, en este caso las temperaturas más bajas se presentan asociadas a situaciones advectivas, con entrada de vientos de componente norte. El aumento de la insolación y radiación recibida durante estos meses en el área de estudio, como en el conjunto de las zonas templadas, y el mayor calentamiento diurno provocan el que descenso de los valores térmicos atribuido a la pérdida de calor nocturna sea menos acusado. Esa es también la causa de que las temperaturas máximas diarias sean más altas que en el resto de las situaciones, al contrario que ocurre en el total de las heladas, donde las que se producen en invierno son las más abundantes y por lo tanto las que determinan las características de las mismas.

Una vez más, en los meses de marzo y abril las situaciones estables son las responsables de que se produzcan heladas. Además, frente al comentario que realizábamos en los párrafos anteriores, vuelven a ser éstas las que registran las temperaturas más bajas, pues durante estos meses la entrada de advecciones frías es muy esporádica 
Tabla 4.- Situaciones de tiempo que han generado heladas de primavera en el observatorio de Zaragoza Aeropuerto (periodo 1972-86, meses de marzo y abril).

Sit. $\mathrm{n}^{\circ}$ veces $\% \mathrm{t}$ media T media HRmax HRmin HRmed Vel.viento

$\begin{array}{lrrrrrrrr}\text { A } & 14 & 51.8 & -1.9^{\circ} & 14.9^{\circ} & 89 & 40 & 64 & 6 \\ \mathrm{~A}+\mathrm{NE} & 3 & 11.1 & -2.1^{\circ} & 13.1^{\circ} & 86 & 53 & 69 & 8 \\ \mathrm{~A}+\mathrm{NW} & 1 & 3.7 & -0.4^{\circ} & 11.8^{\circ} & 79 & 45 & 62 & 0 \\ \mathrm{NE} & 1 & 3.7 & 0.0^{\circ} & 11.4^{\circ} & 74 & 42 & 58 & 14 \\ \mathrm{NW} & 4 & 14.8 & -0.4^{\circ} & 12.3^{\circ} & 78 & 38 & 58 & 17 \\ \mathrm{~W} & 1 & 3.7 & -0.4^{\circ} & 12.0^{\circ} & 88 & 48 & 68 & 7 \\ \mathrm{~B} & 1 & 3.7 & -0.5^{\circ} & 14.3^{\circ} & 83 & 48 & 66 & 4 \\ \text { otras } & 1 & 3.7 & -0.6^{\circ} & 15.4^{\circ} & 63 & 26 & 44 & 25\end{array}$

$\mathrm{t}=$ temp. media de las mínimas diaria; $\mathrm{T}=$ temp. media de las máximas diaria

HRmax; HRmin; HRmed = hum.rel.máxima, mínima y media diarias, en \% vel.viento $=$ velocidad media diaria del viento, a 0 h., en $\mathrm{km} / \mathrm{h}$, a $2 \mathrm{~m}$ de altura

Fuente: I.N.M. Elaboración propia

Es necesario no perder de vista que los umbrales a partir de los que se producen daños en las plantas cultivadas -frutales, sobre todo- en esta época son muy bajos, lo mismo que son muy cortos los períodos temporales que se soportan estas bajas temperaturas, por lo que no es difícil que se superen esos valores críticos. Además hay que tener en cuenta que es precisamente en esta época del año en la que más se deja sentir la influencia de la topografía en los valores térmicos observados. Movernos en las cercanías de estos umbrales supone el que las zonas más expuestas, como las hondonadas y los fondos de valle sufran con frecuencia, aunque a escala reducida, el azote de las heladas tardías, con porcentajes de daños importantes en la cantidad y calidad de las cosechas.

Por todo lo que acabamos de analizar podemos concluir que las olas de frío más rigurosas son, en conjunto, las que presentan un origen advectivo. Su situación más característica está compuesta por un Anticiclón potente situado al oeste de las Islas Británicas y una Baja en Europa meridional o sobre el Mediterráneo. La posición de estos centros favorece la entrada de vientos de componente norte (norte y noreste más frecuentemente, pero también noroeste, aunque en este último caso el descenso de las temperaturas sea algo menor). Naturalmente cuanto mayor es el gradiente de presión existente entre los dos centros de acción que organizan la circulación atmosférica, más intenso es el flujo de vientos que permita el acceso de una masa de aire generalmente ártica y de origen continental. Si además la borrasca se corresponde con inestabilidad. 
en las capas altas de la atmósfera, esa corriente de vientos que penetra sobre el área de estudio se incrementa y si además la temperatura de la atmósfera en altura es baja, alrededor de los 28 grados bajo cero, todo ello tiene como consecuencia que las temperaturas obtenidas en superficie sean excepcionalmente bajas y que la situación de frío sea persistente y duradera. De hecho el episodio frío más riguroso y más largo de toda la serie temporal analizada fue la ola de frío que se produjo en enero de 1985 sucediéndose dos pulsaciones frías, la primera debida al acceso de una masa de aire frío de procedencia ártica, introducida por un flujo de vientos del Norte y por vientos del Noreste la segunda. En el conjunto del valle medio del Ebro se registraron temperaturas excepcionalmente bajas, si bien en los puntos del eje del Ebro y del Jalón, donde la topografía deprimida permitía la acumulación de aire frío en el fondo de los valles, las temperaturas obtenidas fueron más bajas que en las zonas situadas a una altitud algo mayor (HERNANDEZ, en prensa).

Así, con situaciones similares a las que se acaban de describir se consiguen temperaturas mínimas bajas, y a la que se debe añadir la interrupción de la circulación zonal en altura por medio del advenimiento de una situación estable, bien debido al influjo de un anticiclón o de una dorsal anticiclónica, que da lugar a una situación de bloqueo. Como consecuencia, la masa de aire fría que ha llegado hasta esta latitud se estanca y debido al proceso de balance térmico entre la tierra y la atmósfera durante el día y la noche se sobreenfría aún más con lo que los valores térmicos que se registran en los observatorios son todavía más bajos. Hay que tener en cuenta, por otra parte que esta estabilidad que suele seguir a las situaciones de entrada de flujo de componente norte pueden ser debidas, entre otras causas, al ascenso de la presión atmosférica que implica la mayor densidad del aire frío y seco, como también señalan GARCIA DE PEDRAZA, ELIAS CASTILLO y RUIZ BELTRAN (1985). La posición topográfica del área refuerza este proceso térmico facilitando las heladas de irradiación con valores térmicos más bajos.

\section{ALGUNAS CONSIDERACIONES PRACTICAS}

Tal como se señalaba al comienzo de este trabajo, el conocimiento de las situaciones de tiempo que producen heladas permite predecir cuál es su comportamiento, cómo va a ser su desarrollo $\mathrm{y}$, por lo tanto, qué métodos de defensa resultan más económicos y eficaces en este tipo de fenómenos.

Para evitar o reducir los efectos producidos por las heladas se emplean dos métodos de lucha: 
-Lucha directa, cuando se actúa directamente sobre los factores meteorológicos que ocasionan la helada.

-Lucha indirecta, cuando se toman precauciones naturales, con el fin de evitar o reducir al mínimo la influencia de los factores meteorológicos que ocasionan la helada.

Las heladas de irradiación son relativamente fáciles de combatir, tanto por métodos indirectos como directos, puesto que la masa de aire frío se reduce a una capa de aire de poco espesor que está en contacto con el suelo. Por el contrario en las heladas de advección el acceso de la masa de aire fría afecta a un sector espacial de mayor escala y de forma generalizada. A ello hay que añadir el que las situaciones advectivas se presenten acompañadas por vientos fuertes, tal y como ha quedado señalado, que mezclan las masas de aire y homogeneizan las temperaturas. Impedir el descenso de los valores térmicos o aumentarlos es mucho más difícil, pues sería necesaria una infraestructura de predicción de temperaturas, coordinación y combate demasiado costosa; más teniendo en cuenta que este tipo de heladas es mucho menos frecuente que las primeras y que su presencia es sólo testimonial en las primaveras, cuando el riesgo agrícola de daños en cosechas es más alto. Este hecho incrementaría el coste y disminuiría la eficacia.

En realidad, las formas indirectas de lucha contra la helada son las que los agricultores han utilizado tradicionalmente, adaptándose a las potencialidades y limitaciones impuestas por el medio. Simplemente diremos de ellas que consisten en aplicar las condiciones más favorables para el cultivo, como escoger el emplazamiento más adecuado para la instalación de los campos de cultivo. En áreas en las que las heladas tardías presentan un riesgo importante, como es el valle medio del Ebro resulta conveniente elegir las especies y variedades menos vulnerables a los efectos producidos por las heladas, como son las de floración tardía o las que tienen la propiedad de dar una floración posterior sobre la madera del año, que pueden asegurar una cierta cosecha aún en años en que la primera floración ha sido totalmente destruída por una helada.

Por último los daños producidos por temperaturas bajas pueden disminuir si se utilizan las técnicas de cultivo más adecuadas. En este sentido cabe señalar que el suelo y el agua contenida en él poseen una reserva de calor que puede ser cedido a la atmósfera impidiendo así el enfriamiento excesivo de las capas bajas. Esta cesión es tanto más fácil cuando entre el suelo y el aire no se interpone una capa aislante. Se ha comprobado que la diferencia de temperatura, registrada en termómetros situados a 40 $\mathrm{cm}$ de altura, entre un campo cuyo suelo esté apelmazado y húmedo y otro recién labrado puede ser de 1 a $2^{\circ}$ y que la diferencia entre aquél y uno con cubierta vegetal puede llegar incluso a $2.5^{\circ}$ (GESLIN, 1963; FUENTES, 1978). Por ello, en el caso de las 
plantaciones de frutales resulta conveniente mantener el suelo desnudo, sin vegetación y evitar remover la tierra. De la misma forma es fundamental procurar una buena polinización y realizar una protección fitosanitaria muy cuidadosa. Así se puede aumentar la resistencia de las plantas proporcionándoles las mejores condiciones de alimentación y sanidad.

No obstante, estos sistemas de lucha indirecta o de adaptación no siempre se utilizan, y en ocasiones, buscando un mayor beneficio económico, se implantan variedades nuevas de mayor rendimiento pero más susceptibles de ser afectadas por las heladas de primavera, por lo que es necesario utilizar métodos directos o activos.

Los métodos de lucha directos están encaminados a retrasar la entrada en determinados estados fenológicos de los vegetales o bien a mantener la temperatura del vegetal por encima del nivel crítico, por medio de la aplicación de diversas técnicas. Entre ellas destacan la aplicación de procedimientos bioquímicos, el recubrimiento de las plantas, la formación de humos y nieblas artificiales, la puesta de marcha de ventiladores, estufas y quemadores, la aplicación de rayos infrarrojos, o el riego por aspersión. El efecto producido por cada uno de ellos es variable, por lo que para la aplicación de unos u otros resulta conveniente el conocimiento de los procesos de formación de heladas, su desarrollo y frecuencia de aparición puesto que el costo de la protección y el número de intervenciones necesarias evoluciona de forma muy diferente según cuál sea el procedimiento utilizado y la superficie a proteger. No entraremos en la explicación detallada de cada uno de ellos, puesto que no es nuestra intención, sino que simplemente indicaremos algunas ideas a este respecto, en relación con el análisis de la formación de heladas.

El uso de los ventiladores puede ser eficaz en el caso de las heladas por irradiación cuando sea necesario un aumento de temperatura que oscile entre 1 y $2^{\circ}$. Este método aumenta al combinarse con el de calefacción, que puede conseguir un aumento de los valores térmicos de 2 a $4^{\circ}$.

En este sentido cabe destacar la experiencia llevada a cabo en los años 80 por un grupo de agricultores del campo de la Almunia, en el valle del Jalón (provincia de Zaragoza) (GUZMAN, 1988), en un área muy cercana al emplazamiento del observatorio utilizado en este análisis y con características climáticas muy similares (HERNANDEZ, 1992). En este área, debido a la implantación de variedades frutales de floración más temprana que las tradicionales, aumentaron los daños producidos por las heladas, traducidas en pérdidas importantes de la cosecha y por lo tanto en el aumento de las tasas de los seguros agrarios, ya que las indemnizaciones llegaron en algunos años a superar a las primas pagadas. Por ello las organizaciones profesionales agrarias, apoyadas por la Administración y las entidades aseguradoras establecieron un sistema de defensa contra heladas. 
El sistema que ha proporcionado mejores resultados ha sido el de las torres ventilador. Con este método, de potencias variables entre los 25 y 200 C.V. se pueden ganar hasta $2^{\circ}$ en el nivel de los órganos a proteger. Esta cifra puede sufrir variaciones, pero se suele aceptar como indicativa para su utilización en la práctica. Es el más efectivo precisamente porque la mayor parte de las heladas que se producen en esta zona son de irradiación, ya que este sistema no puede utilizarse en la defensa contra las heladas de advección o de evaporación, si bien en este último tipo de heladas, durante el funcionamiento de la torre-ventilador, al no depositarse sobre los órganos vegetales ni rocío ni escarcha, se evita el enfriamiento por evaporación.

\section{CONCLUSIONES}

El análisis de los episodios de helada observados en el centro de la Depresión del Ebro permite concluir que la mayor parte de ellas están ocasionadas por una situación de estabilidad atmosférica, más intensa y perceptible en las capas bajas en contacto con la superficie, aunque puedan verse alimentadas por la entrada de aire frío -de componente norte- en altura. Estas situaciones se caracterizan por presentar valores altos de presión en superficie, superiores a los $1.020 \mathrm{mb}$, y la temperatura de las capas altas oscila en torno a los $-20^{\circ} /-24^{\circ}$.

Las heladas asociadas a situaciones estables, además de ser las más frecuentes y las de mayor duración en conjunto son también las que producen los valores de temperatura más bajos, tanto en las mínimas como en las máximas. Sólo durante la primavera, las temperaturas más bajas se producen asociadas a situaciones advectivas, aunque el mayor porcentaje de heladas se deba a la irradiación.

Las heladas de advección son menos frecuentes, aunque la entrada de una pulsación fría -generalmente acompañada de vientos fuertes- y posterior establización de la masa de aire y génesis de valores térmicos aún más bajos se repite frecuentemente en las situaciones de helada.

Como consecuencia de todo lo anterior podemos concluir que la influencia del sustrato geográfico y de la topografía es muy importante, tanto como la evolución atmosférica, en la génesis de las heladas en el valle medio del Ebro. Este fenómeno es el responsable de que sea la fuerte irradiación nocturna asociada a situaciones estables, anticiclónicas, la que genere temperaturas en superficie inferiores a $\operatorname{los} 0^{\circ}$, en mayor proporción que en el resto de los tipos de tiempo. En definitiva, las heladas más rigurosas en conjunto son las que presentan un origen doble o combinado, con entrada de una advección fría y posterior establización de la masa de aire. 
Por otro lado, para que la lucha contra las heladas pueda ser lo más eficaz posible, y debido a que la mayor parte de las heladas que se generan en el valle medio del Ebro están ligadas a una situación estable, resultaría muy necesario disponer de índices actinotérmicos en la red de observatorios meteorológicos que permitan establecer los gradientes verticales que existen entre los valores de las temperaturas observados en garita y los realmente soportados por las plantas.

\section{BIBLIOGRAFIA}

CAÑADA TORRECILLA, M.R. (1990): "Diferencias estacionales entre tipo de tiempo ciclónico y anticiclónico en Extremadura", Anales de Geografía de la Universidad Complutense, 10, 85-102.

DE FINA Y RAVELO (1979): Climatología y fenología agrícolas, Eudeba, 352 p.

FUENTES YAGÜE, J.L.(1978): Apuntes de Meteorología agrícola, Ministerio de Agricultura, $314 \mathrm{p}$.

GARCIA DE PEDRAZA, L., ELIAS CASTILLO, F. Y RUIZ BELTRAN, L. (1985): Estudio de heladas en España, I.N.M., publicación A-76, 52 p.+mapas.

GUZMAN CORDOBA, J.A. (1988): "Sistema de defensa contra heladas en la comarca de La Almunia", Surcos, 7, 4-39.

HERNANDEZ NAVARRO, M.L.(1992): Climatología agrícola del valle medio del Ebro (sector central de la Depresión), Dpto. de Geografía y Ord. del Territorio, Univ. de Zaragoza, (tesis doctoral inédita), $1.351 \mathrm{p}$.

HERNANDEZ NAVARRO, M.L. (en prensa): El riesgo de helada en plantaciones frutales, Institución "Fernando el Católico", Diputación Provincial de Zaragoza.

O.M.M. (1983): Guía de prácticas climatológicas, O.M.M., 100, TP 44.

URBANO, P. (1989): Tratado de Fitotecnia general, Ed. Mundi-Prensa, 836 p. 